\title{
The Collective Behaviors of Self-Excitation Information Diffusion Processes for a Large Number of Individuals
}

\author{
Lifu Wang ${ }^{1}$ and Bo Shen (D) $^{2}$ \\ ${ }^{1}$ Department of Electronic and Information Engineering, Beijing Jiaotong University, Beijing, China \\ ${ }^{2}$ China Key Laboratory of Communication and Information Systems, \\ Beijing Municipal Commission of Education Beijing Jiaotong University, Beijing, China \\ Correspondence should be addressed to Bo Shen; bshen@bjtu.edu.cn
}

Received 25 June 2020; Accepted 24 July 2020; Published 24 August 2020

Guest Editor: Hongshu Chen

Copyright (C) 2020 Lifu Wang and Bo Shen. This is an open access article distributed under the Creative Commons Attribution License, which permits unrestricted use, distribution, and reproduction in any medium, provided the original work is properly cited.

\begin{abstract}
The opinion dynamics is a complex and interesting process, especially for the systems with a large number of individuals. It is usually hard to describe the evolutionary features of these systems. In some previous works, it has been shown that the selfexcitation type model has superior performance in learning and predicting opinions. Following this line, we consider the selfexcitation opinion model and study the collective behaviors of the self-excitation model. We propose a Mckean-Vlasov-type integrodifferential equation to describe the asymptotic behaviors of the model and show that the introduced equation, by coupling with the initial distribution, has the ability of capturing the influence of the self-excitation process, which describes the mutually exciting and recurrent nature of individuals. We also find that the steady-state distribution is a "contraction" of the initial distribution in the linear and bounded confidence (DW model) interaction cases, which is different from the results of the model with nonself-excitation interaction.
\end{abstract}

\section{Introduction}

Expressing opinions and then influencing others are the primary forms of social behavior of people. The dynamics of public opinion has long been a major concern of social science and computational social science. There has been an increasing interest on the perception and prediction of social opinion, such as quantitative investment firms which measure investor sentiment and trade using social media Karppi and Crawford [1] and prediction of election results [2].

Based on sentiment analysis with deep learning, there are many frameworks to perceive opinion on the social network. For the prediction of social opinion, there are some noticeable models proposed at the beginning of the $21^{\text {st }}$ century, such as the Sznajd model [3], Hegselmann-Krause model [4, 5], Deffuant-Weisbuch model [6], and so on, which had been established to understand the co-ordinated movements of opinion as a group. However, these works held some limitations: (i) most of these models on opinion dynamics are theoretical and have not proved their effectiveness quantificationally. (ii) These models are represented by a cellular automaton, which makes it difficult to analyze the global behavior of the dynamics.

More recently, some researchers began to study the models, which can provide more accurate predictions [7-9]. De et al. [9] proposed a framework of opinion dynamics named SLANT, providing accurate predictions of users' opinions. In the early models, the opinion and the states of the next moment only depend on the states of the last moment, and thus have nothing to do with more previous ones. It means that opinion formation is seen as a Markov process. However, in the real world, this assumption may not be precise. The model given in the study by $\mathrm{DE}$ et al. [9] indicates that self-excitation opinion models with exponential function weight have superior performance in learning and predicting opinions. 
In many works [9-13], self-excitation models have shown efficient abilities to capture users' behavior pattern. These models focus on the microbehaviors basing on cellular automata, which would not express the global dynamics of opinion evolution analytically.

In this paper, we study the self-excitation opinion dynamics on the large homogeneous network. We show that when the number of the individuals is very large, the opinion distribution of this system evolves according to a nonlinear partial differential equation (PDE) of Mckean-Vlasov type. Due to the dependence on the initial value in the local rules, the individuals with different initial values will have different dynamic equations; thus, the mean-field equation deriving process is different from the cases of the previous works $[14,15]$. We show that this PDE can be decomposed into two parts where the first part is the same as the Fokker-Planck model in the study by Toscani [16]. And the second part can capture the mutually exciting and recurrent nature of individual behaviors by an integral term related to the initial value. This term leads to different steady state behaviors from the Markov model, such as the model given in the study by Toscani [16]. Generally, the stationary solutions of the Toscani type model will trend to a single-point support Dirac measure when noise parameters tend to 0 , but the model with additional term makes the steady state to be a "contraction" of the initial distribution.

The rest of this paper is organized as follows. Section 2 is for related work. In Section 3, we introduce the mathematical model of opinion dynamics we study and the main mean-field theorem in this paper. In Section 4, using this theorem, we consider the linear interaction model and the bounded confidence (DW) model and study the steady-state distribution of these models. Section 5 is the proof outline of this theorem. Section 6 is a summary. The detailed proof is in the appendix.

\section{Related Work}

2.1. Information Diffusion Model. The early theory of information diffusion in mathematical sociology was proposed by Sznajd in the study by Sznajd-Weron and Sznajd [3]. Sznajd's model is designed to explain the features of opinion dynamics, in which every individual is on a lattice and have a state 0 or 1 to express the opinion. Individuals update their opinion by the opinions of their neighbors. The numerical simulations of this model are also investigated by many researchers [17-19]. On the complete graph, Slanina and Lavicka [20] show that when the number of individuals is very large, the probability densities evolve according to the partial differential equations

$$
\begin{aligned}
& \frac{\partial}{\partial \tau} P(m, \tau)=\frac{\partial^{2}}{\partial m^{2}}\left[\left(1-m^{2}\right) P(m, \tau)\right], \\
& \frac{\partial}{\partial \tau} P(m, \tau)=-\frac{\partial^{2}}{\partial m^{2}}\left[\left(1-m^{2}\right) m P(m, \tau)\right],
\end{aligned}
$$

where $P(m)$ is the probability distribution of opinion. Equation (1) describes the Ochrombel simplification of the
Sznajd model, and (2) does the original case. The authors showed the existence of phase transition in the original formulation and smooth behavior without transition in the model modified by Ochrombel.

For the theory of continuous opinion dynamics, the Deffuant-Weisbuch model [6] and Hegselmann-Krause model $[4,5]$ deal with two different cases. Both these models are so-called bounded confidence models, i.e., the individuals can only be influenced by some others whose opinions are close enough to theirs. The characteristics of bounded confidence lead to the formation of communities, which coincides with the phenomenon in the real world. A very beautiful analysis of the convergence of the Deffuant-Weisbuch model is given in the study by Zhang et al. [21], which reveals the community structure of the model. Bhattacharyya [22] proves the convergence of the Hegselmann-Krause model. On the other hand, numerical simulations on complex networks [23] show that "the more heterogeneous the complex network is, the weaker the ability of polarization and consensus of the complex network will be." Models with both continuous opinion and discrete states are also considered, such as the SHIR model [24], where the individuals have a four-state variable to represent susceptible, hesitated, infected, and removed. Opinion dynamics of the social network and the impact on the recommendation are studied in the studies by Xiong et al. [25] and $\mathrm{Li}$ et al. [26].

Como and Fagnani [27] gave a general framework to prove that the scaling limits of the many-body continuous opinion system can be described by a measured value ordinary differential equation (ODE). In such models, every individual will change its opinion to $\omega X+(1-\omega) X^{\prime}$, a convex combination with the neighbor with probability $k\left(X, X^{\prime}\right)$. When $k\left(X, X^{\prime}\right)=1_{[0, \varepsilon]} \mid\left(\left|X-X^{\prime}\right|\right)$, it becomes the Deffuant-Weisbuch model. The key ideas of their work are the large deviation principle and some estimations based on the Lipschitz property, which are the same as the general method used to prove the chaotic propagation properties [28].

Based on the Boltzmann equation with granular gas like interactions, Toscani [16] introduced a collision model, in which the communication between individuals was considered as a collision with the following form:

$$
w^{\prime}=\left(1-\eta P\left(w, w_{*}\right)\right) w+\eta P\left(w, w_{*}\right) w_{*}+\zeta D(w)
$$

where function $D$ and $P$ describe the local relevance of the compromise and diffusion for a given opinion. Toscani also considered the quasi-invariant opinion limit for the Boltzmann-type equation. The main idea of that is to scale the interaction frequency, strength, and the diffusion in the integral equation, and then, the equation reduces to a Fokker-Planck type equation. Then, on the base of this model, considering the influence of the structure of the social network, Albi et al. [29] consider the kinetic opinion dynamics on the large scale networks evolving over time. The evolutions of both the network and the opinion were involved. 
2.2. Self-Excitation Point Process in Social Systems. Point processes type models are generally used to analyze the impact of events on the system such as CSMA/CD [30], financial data [31], and the dynamics of book sales [32]. A very succinct and effective analysis for the time series of daily views for videos on YouTube is introduced [33]. The authors showed that most of the video viewing record data could be described statistically by a Poisson process, but still, about $10 \%$ data show a point process with the instantaneous rate $\lambda(t)$,

$$
\lambda(t)=V(t)+\sum_{i, t_{j} \leq t} \mu_{i} \phi\left(t-t_{i}\right),
$$

where $\mu_{i}$ is the number of potential viewers influenced directly by person $i$ who views the video through the social network, and $V(t)$ captures all spontaneous views that are not triggered by epidemic effects on the network.

For the self-excitation social opinion models, De et al. [9] proposed an opinion model on a social network, in which every user has a latent opinion about the given topic and can post messages about the topic. The experiments on real data show that the self-excitation model, with the intensity depending on the messages sent from a neighbor in the past and the weight function having the form like $e^{-\omega t}$, performs much more better than the Poisson process $(\omega=0)$ model.

Wang et al. [13] used point process to predicting user activities. They proposed a generic framework for point process prediction problem, and they used a mass transport equation to update the transport rate and compute the conditional mass function.

2.3. Mean Field Theory. Mean-field theory (MFT) is introduced to study many-body problem by using a single averaged effect to approximate the effect of all individuals. The core of MFT is to estimate the error of the mean-field approximation. If there is no interaction, all individuals are independent of each other, and then, the law of large numbers works and gives the mean-field approximation. In the general case, we still want to be able to use the law of large numbers. That is to say, if one picks a chaotic (i.i.d) initial distribution of particles, we hope that this distribution is still chaotic as time-evolving, which is the so-called propagation of chaos.

The theory of propagation of chaos in the cases of Wiener noise is summed in the study by Sznitman [28]. If $N$ particles with initial "chaotic" distribution $u_{0}^{\otimes N}$ satisfy the stochastic differential equation (SDE),

$$
\mathrm{d} X_{i}=\mathrm{d} W_{i}+\frac{1}{N} \sum_{j} b\left(X_{i}, X_{j}\right) \mathrm{d} t
$$

the Mckean-Vlasov mean-field equation is

$$
\mathrm{d} X=\mathrm{d} W+\int b(X, y) u(\mathrm{~d} y) \mathrm{d} t,
$$

where $u$ is the law of $X$. The method to show this is to use the Lipschitz character of $b$ and Gronwall's lemma. Mckean-Vlasov equation for SDE with Poisson jumps is in the study by
Andreis et al. [15]. The authors used Burkholder-Davis-Gundy inequality for martingales. The main step in the study by Andreis et al.[15] is to use Doob-Meyer decomposition and estimate the compensated Poisson process.

Using the large deviation principle [34], Arous and Guionnet [35] studied the mean-field simplification dynamics for Langevin spin glass. The framework of their proof makes use of Sanov's theorem and Varadhan's lemma in the path space $C[0, T]$ to get the rate function for the interaction system.

The main obstacle to use these methods to prove MFT is the unboundedness of coefficients in SDE. Because of Varadhan's lemma, if we use a stop time to get the localization of SDE, we can get the so-called local large deviation principle. Dawsont and Gartner [36] gave some compactness criteria to convert the "local" result into "global" one. They also show that a Lyapunov function for the system of weakly interacting diffusions will let the compactness condition be satisfied. Puhalskii [37] set up the whole framework of local to global LDP and introduce the $C$-exponential tightness conditions.

\section{Mathematical Model}

3.1. Self-Excitation Opinion Model. In this paper, we consider a simplified version of the model [9] and study the large number limit of the self-excitation opinion model with the following form:

There are $N$ individuals with opinion $x_{i}$, which is a function of the time $t$, such that

$$
x_{i}(t)=x_{i}(0)+\sum_{j} h\left(x_{j}, x_{i}\right) k(t) * \mathrm{~d} N_{j}(t),
$$

where $h\left(x_{j}, h_{i}\right)$ is a interaction function, $*$ is the convolution operator, $k(t)=e^{-\omega t}$ is the triggering kernel, and $N(t)$ is a Poisson process. This model has been previously studied $[11,12]$.

The original model proposed in the study by De et al. [9] has the following form:

$$
\begin{aligned}
x_{i}(t) & =b_{i}+\sum_{j} \alpha_{i, j} \sum_{t_{j} \in H_{j}(t)} m_{j} k\left(t-t_{j}\right) \\
& =b_{i}+\sum_{j} \alpha_{i, j} m_{j} k(t) * \mathrm{~d} N_{j}(t),
\end{aligned}
$$

where $m_{j}$ is the message sent by user $j, \alpha_{i j}$ is the interaction intensity between $i$ and $j, b_{i}=x_{i}(0)$ is the initial opinion of user $i, H_{j}(t)$ is the history of events up to time $t$ for user $j$, and $k(t)$ is a triggering kernel. The values of message $m$ come from a sentiment distribution related to the opinion $x$ and $E\left[m_{i} \mid x_{i}\right]=x_{i}$.

As the models have been previously studied $[11,12]$, we only consider the opinion part, the simplified model (7). In the case that $h\left(x_{i}, x_{j}\right)=h\left(x_{j}\right)$, where $x_{j}$ is the opinion of the individual $j$, De et al. and Wang et al. $[9,11]$ showed the validity of the model in the study by DE et al. [9] on the real world data. And when $h\left(x_{j}, x_{i}\right)=x_{j}-x_{i}$, the form of this model will degenerate into the form of the DW model [6] and Toscani model [16] when $k(t)=1$. 
In studies by De et al. and Wang et al. $[9,11]$, the selfexcitation opinion model with exponential function type weight was shown to be very effective in the opinion dynamics; therefore, we set $k(t)=e^{-\omega t}$, where $\omega$ is a constant. Due to the fact that differential of the convolution of two functions $d(f * g)=f(0) g+g * d f$, where there is a jump part in $g$, and $\mathrm{d} k(t)=-\omega k(t) \mathrm{d} t$. We can use a stochastic differential equation (SDE) to describe $x(t)$ [11].

$$
\mathrm{d} X_{i}=\omega\left(b_{i}-X_{i}(t)\right) \mathrm{d} t+\sum_{j} \alpha h\left(X_{j}, X_{i}\right) \mathrm{d} N_{j} .
$$

It is also necessary to add $\sigma \mathrm{d} W$ into the model to represent noise, where $W$ is the Wiener noise with $W[0]=0$.

$$
\mathrm{d} X_{i}=\omega\left(X_{i}(0)-X_{i}(t)\right) \mathrm{d} t+\sum_{j} \alpha h\left(X_{j}, X_{i}\right) \mathrm{d} N_{j}+\sigma \mathrm{d} W_{i} .
$$

The above two equations are given in the study by Wang et al. [11]. Because of the dependence on the path of the history, it is apparently not a Markov process. We consider the limit that the population size tends to infinity. Let $X^{N}=$ $\left(X_{1}, X_{2}, \ldots, X_{N}\right)$ be the opinion variable of the $N$-particles system. $X$ satisfies

$$
\mathrm{d} X_{i}=\omega\left(b_{i}-X_{i}(t)\right) \mathrm{d} t+\frac{1}{N} \sum_{j \neq i}^{N} \alpha h\left(X_{j}, X_{i}\right) \mathrm{d} N_{j}+\sigma \mathrm{d} W_{i}
$$

where $b_{i}=X_{i}[0]$. In the case that $\omega=0$ and $h(x, y)=$ $(x-y) 1_{[0, \varepsilon]}(|x-y|)$, this is a Deffuant-Weisbuch-like model.

If we only consider the case $\omega=0$ and replace the Poisson Noise $\mathrm{d} N$ with $\mathrm{d} t$ and consider the form

$$
\mathrm{d} X_{i}=\mathrm{d} W_{i}+\frac{1}{N} \sum_{j} h\left(X_{i}, X_{j}\right) \mathrm{d} t
$$

The mean-field theory of this equation is well studied [28]. The Mckean-Vlasov mean-field equation is

$$
\mathrm{d} X=\mathrm{d} W+\int b(X, y) u(\mathrm{~d} y) \mathrm{dt} .
$$

In this paper, we first use Doob-Meyer decomposition for $\mathrm{d} N$. Then, we estimate the compensated Poisson process by Burkholder-Davis-Gundy inequality for martingales. This method helps us to reduce our equation to the form in (12). As for the dependence on the initial values, we regard the initial as a random media. Using the methods in the study by Pra and Hollander [38]. The Mckean-Vlasov equation can be obtained using Sanov's theorem and Varadhan's lemma in the path space $C(0, T)$. In this paper, we use a direct estimation to prove the convergence to the Mckean-Vlasov equation.

3.2. Main Results. The main result in this paper is the following theorem:

Theorem 1. When $N$ tends to infinity, there is a limit process $X$ for $X^{N}$ in equation (11), with SDE:

$$
\mathrm{d} X=\int \mathrm{d} P(y) h(y, X) \lambda \mathrm{d} t+\omega(b-X) \mathrm{d} t+\sigma \mathrm{d} W
$$

where $P$ is the law of $X$, and $b=X[0]$ is a random variable. And given the initial distribution $\mu$, the law of $X$ is $P=\int P^{b} d \mu(b)$, where $P^{b}$ is the solution of the Fokker-Planck equation associated with the Mckean-Vlasov process:

$$
\begin{aligned}
\frac{\partial}{\partial t} P^{b} & =-\frac{\partial}{\partial x}\left[\beta^{b, P} P^{b}\right]+\frac{1}{2} \sigma^{2} \frac{\partial^{2}}{\partial x^{2}} P^{b}, \\
P^{b}(0) & =\delta_{b}, \\
\beta^{b, P} & =\int \mu\left(\mathrm{d} b^{\prime}\right) \int P^{b^{\prime}}(\mathrm{d} y) \alpha \lambda h(y, x)+\omega(b-x),
\end{aligned}
$$

where $\delta_{b}$ is a Dirac measure with support on $b$.

From this theorem, we can study the asymptotic behavior of the self-excitation opinion model. In Section 4, we will study the steady state of this system by using Fokker-Planck equation and show the effects of self-excitation, which makes it impossible to give a perfect consensus, such that it can avoid Abelson's diversity puzzle [39].

In order to prove this theorem, we need some assumptions. Stemming from the physical meaning, we can assume that the support of initial distribution $\mu(b)$ is bounded, such as the uniform distribution on $[0,1]$. Also, for the proof of Mckean-Vlasov limit, we need the function $h(x)$ to have good enough properties to make

$$
\begin{gathered}
E\left[h\left(X_{j}^{b}, X_{i}^{b}\right)\right]<\infty, \\
\int x^{2} P(\mathrm{~d} x)<\infty,
\end{gathered}
$$

where $P$ is the solution of the Mckean-Vlasov equation.

If $h$ is bounded, the first condition is satisfied trivially. In the appendix, we will give other examples of $h$ that can make these conditions be satisfied. Also, we assume that $h(x, y)$ is Lipschitz for both of the variables. However, this is not the necessary condition [14].

$$
\left|h\left(x_{1}, y_{1}\right)-h\left(x_{2}, y_{2}\right)\right|<L\left|x_{1}-x_{2}\right|+K\left|y_{1}-y_{2}\right| \text {. }
$$

Most of these conditions can be relaxed, but we hope to prove our results without excessive technical details on tightness; therefore, these restrictions are imposed.

\section{The Properties of Mckean-Vlasov Dynamics}

4.1. Mckean-Vlasov Equation. For the equation

$$
\begin{aligned}
\frac{\partial}{\partial t} P^{b} & =-\frac{\partial}{\partial x}\left[\beta^{b, P} P^{b}\right]+\frac{1}{2} \sigma^{2} \frac{\partial^{2}}{\partial x^{2}} P^{b}, \\
P^{b}(0) & =\delta_{b}, \\
\beta^{b, P} & =\int \mu\left(\mathrm{d} b^{\prime}\right) \int P^{b^{\prime}}(\mathrm{d} y) \lambda h(y, x)+\omega(b-x),
\end{aligned}
$$

we consider a simple case 


$$
\begin{aligned}
\lambda & =1, \\
\sigma & =1, \\
h(y, x) & =\alpha(y-x) .
\end{aligned}
$$

Then, we can see that

$$
\beta^{b, P}=\alpha(m-x)+\omega(b-x),
$$

where $m=\int x \mathrm{~d} P(x)$, the mean value of $x$.

Since $h$ is an odd function, it is easy to see that

$$
\begin{aligned}
\frac{\mathrm{d} E(X(t))}{\mathrm{d} t} & =E\left(\left[\alpha \int P(\mathrm{~d} Y)(Y-X)+\omega(b-X(t))\right]\right) \\
& =0+\omega E[X(0)-X(t)] .
\end{aligned}
$$

So, $E(X(t)-X(0))=C e^{-\omega t}$ since $E(X(0)-X(0))=0$, $C=0$, and $m=E(X(t))=E(X(0))$.

Then, we have the Fokker-Planck equation associated with the Mckean-Vlasov process:

$$
\frac{\partial}{\partial t} P^{b}=-\frac{\partial}{\partial x}\left[\alpha(m-x)+\omega(b-x) P^{b}\right]+\frac{1}{2} \frac{\partial^{2}}{\partial x^{2}} P^{b}
$$

Integral over $\mu(b)$

$\frac{\partial}{\partial t} P=-\frac{\partial}{\partial x}\left[(\alpha(m-x)) P+\omega \int d \mu(b)(b-x) P^{b}\right]+\frac{1}{2} \frac{\partial^{2}}{\partial x^{2}} P$.

Another example is the bounded confidence type model $[4,6]$ where every agent interacts only within a certain level of confidence:

$$
h(y, x)=(y-x) k(y-x),
$$

where $k(y-x)$ is a continuous function, and if $y-x<\Delta_{1}, k(y-x)=1$, and if $y-x>\Delta_{2}, k(y-x)=0$. Since, in this case, $h(x)$ is bounded and Lipschitz, our assumptions are satisfied.

Note that for the case that $h(y, x)=\alpha y$, which is used in the SLANT system [9],

$$
\frac{\partial}{\partial t} P^{b}=-\frac{\partial}{\partial x}\left[\alpha m(t)+\omega(b-x) P^{b}\right]+\frac{1}{2} \sigma^{2} \frac{\partial^{2}}{\partial x^{2}} P^{b},
$$

where $m(t)=\int x \mathrm{~d} P(x, t)$. Since

$$
\begin{aligned}
\frac{\mathrm{d} E(X(t))}{\mathrm{d} t} & =E\left[\alpha \int P(\mathrm{~d} Y) Y+\omega(b-X(t))\right] \\
& =(\alpha-\omega) E(X)+\omega E(X(0)) .
\end{aligned}
$$

As a sanity check, this formula matches (4) in the study by De et al. [9]. Solving this equation, we get

$$
\begin{aligned}
m(t)+\frac{\omega}{\alpha-\omega} m(0) & =C e^{(\alpha-\omega) t}, \\
C & =\frac{\alpha}{\alpha-\omega} .
\end{aligned}
$$

4.2. Discussion. Como and Fagnani [27] showed that when the population size tends to infinity, the limit behavior of the Deffuant-Weisbuch model can be described by a measurevalued ODE. And also, Toscani introduced a kinetic model of opinion formation [16]. In this model, the opinion is changed by the binary interaction of collision, and the dynamics of the opinion distribution is modeled by Boltzmann-type integrodifferential equation.

In these models, the dynamics of opinion distribution is depicted as follows:

$$
\frac{\mathrm{d}}{\mathrm{d} t}\left\langle\phi, \mu_{t}\right\rangle=\left\langle\phi, H\left(\mu_{t}\right)\right\rangle
$$

where $\mu_{t}$ is the measure of opinion distribution, and $\phi$ is an arbitrary test function. The right side of (29) has the following form [27]:

$$
\left\langle\phi, H\left(\mu_{t}\right)\right\rangle=\iint(\phi(1-\omega) x+\omega y)-\phi(x) k(x, y) \mathrm{d} \mu(x) \mathrm{d} \mu(y),
$$

and in Boltzmann case [16],

$$
\begin{aligned}
\left\langle\phi, H\left(\mu_{t}\right)\right\rangle & =\iint \phi\left(w^{\prime}\right)+\phi\left(w_{*}^{\prime}\right)-\phi(w)-\phi\left(w_{*}\right) \mathrm{d} \mu(w) \mathrm{d} \mu\left(w_{*}\right), \\
w^{\prime} & =1\left(-\eta P\left(w, w_{*}\right)\right) w+\eta P\left(w, w_{*}\right) w_{*}, \\
w_{*}^{\prime} & =\left(1-\eta P\left(w_{*}, w\right)\right) w+\eta P\left(w_{*}, w\right) w .
\end{aligned}
$$

Intuitively, both models can be derived from the stochastic differential equation with Poisson jump interactions by the method mentioned in the study by Méléard [14]. Events that interact with other particles are regarded as a Poisson process.

For the system with $h(y, x)=y-x$,

$$
\frac{\partial}{\partial t} P=-\frac{\partial}{\partial x}\left[(\alpha(m-x)) P+\omega \int \mathrm{d} \mu(b)(b-x) P^{b}\right]+\frac{1}{2} \frac{\partial^{2}}{\partial x^{2}} P,
$$

in the case that $\omega=0$, which is the nonself-excitation case, it degenerates into the general opinion dynamics model, or equally, the quasi-invariant opinion limit for Toscani's Boltzmann equation [16]:

$$
\frac{\partial}{\partial t} P=\frac{\lambda}{2} \frac{\partial^{2}}{\partial x^{2}}\left(D\left(|x|^{2}\right) P\right)-\frac{\partial}{\partial x}(P(|x|)(m-x) P) .
$$

The $\omega$ term is a modification to consider the influence of the self-excitation process.

In this paper, we only consider the homogeneous network. However, using the method in the study by Pra and Hollander and Albi et al. [38, 40], we can study the large system of agents interacting through a network with a given distribution of the number of connections by the following way:

(1) Consider a random media $c \in\left\{0,1,2, \ldots, c_{\max }\right\}$, which is a discrete variable describing the number of connections. $c$ should be a random variable to 
represent the complex network. For example, $c$ obeys power law distribution.

(2) Construct a new interaction equation as in the study by Albi et al. [40].

$$
\mathrm{d} X_{i}=\omega\left(b_{i}-X_{i}(t)\right) \mathrm{d} t+\sum_{j} \alpha h\left(X_{j}, X_{i}, c\right) \mathrm{d} N_{j} .
$$

Then, it is the same as Theorem 1 in the study by Pra and Hollander [38] such that in this random media, we can also prove a mean-field theorem. And there is a distribution $P(c, x, t)$ about the variable $c$, opinion $x$, and time $t$. Using a similar argument as Albi et al. [40], we have obtained the stationary solutions.

4.3. Steady-State Distribution of the Linear Case. Considering a simple case of initial distribution $P_{0}=1 / 2 \delta_{-10}+1 / 2 \delta_{10}$. We will use this simple form to explain how the initial distribution is coupled to the equation. Then, the equation will become

$$
\begin{aligned}
\frac{\partial}{\partial t} P^{\{-10\}}= & -\frac{\partial}{\partial x}\left[(\alpha(\bar{x}-x)) P^{\{-10\}}+\omega(-10-x) P^{\{-10\}}\right] \\
& +\frac{1}{2} \frac{\partial^{2}}{\partial x^{2}} P^{\{-10\}}, \\
\frac{\partial}{\partial t} P^{\{10\}}= & -\frac{\partial}{\partial x}\left[(\alpha(\bar{x}-x)) P^{\{10\}}+\omega(10-x) P^{\{10\}}\right] \\
& +\frac{1}{2} \frac{\partial^{2}}{\partial x^{2}} P^{\{10\}} .
\end{aligned}
$$

The overall distribution $P$ is equal to $1 / 2 P^{\{-10\}}+1 / 2 P^{\{10\}}$. The evolution process of this distribution is shown in in Figure 1(a). And Figures 1(b) and 1(c) show the differences between two kinds of steady states.

As shown in Figure 1(b), for the initial distribution $P_{0}$, the steady state distribution is normal. In fact, this is true for any initial distribution, and we have the following theorem.

Theorem 2. In the case that $\omega=0$, for all type of initial distribution $P_{0}$, we have the steady-state distribution $P(\infty)=$ $1 / Z e^{(\alpha(x-m) / \sigma)^{2}}$, where $Z$ is the normalization constant.

This is because the form of PDE for $P$ has the form

$$
\frac{\partial}{\partial t} P=-\frac{\partial}{\partial x}(\alpha(m-x)) P+\sigma^{2} \frac{1}{2} \frac{\partial^{2}}{\partial x^{2}} P .
$$

The steady-state equation

$$
-\frac{\partial}{\partial x}(\alpha(m-x)) P+\sigma^{2} \frac{1}{2} \frac{\partial^{2}}{\partial x^{2}} P=0,
$$

and this steady state is asymptotically stable, such that when $t \longrightarrow \infty$, the solution of the equation will trend to the steady state. It is easy to see that the steady state is a normal distribution as shown in Figure 1(b).
From Theorem 2, this $\omega=0$ model cannot describe the actual situation since, in this model, the final state of the opinion distribution has no community structures but a perfect consensus (in fact, this is true for any monotonic function $h$, since the steady state is the Gibbs distribution of a potential energy $V(x)$, such that $\nabla V=h$ ), which is so-called Abelson's diversity puzzle [39], a persistent research puzzle in the social sciences. Generally, this puzzle is solved by the bounded-confidence mode, such as HK [4] and DW [6] models. It is proved in the study by Como and Fagnani [27] for a large number of individuals case and by Zhang et al. [21] for the general case that the steady state of the DW model has the form $\sum_{i} C_{i} \delta_{x_{i}}$, where $x_{i}$ is the opinion of the community $i$, and $\mid x_{i}-$ $x_{j} \mid>R$ if $i \neq j$, where $R$ is the bounded confidence distance.

However, in the case $\omega>0$, the equation has the steady state as in Figure 1(c) when the initial distribution has the form $1 / 2\left(\delta_{-10}+\delta_{10}\right)$. In the general case, we have

Theorem 3. In the case that $\omega>0$, given an initial distribution $\mu(x)$, we have the steady-state distribution $P(x, \infty)=1 / Z \int \mu(b) e^{-((\alpha+\omega) x-(\alpha m+\omega b) / \sigma)^{2}}$, where $Z$ is the normalization constant.

Let $P=\int \mathrm{d} \mu(b) P^{b}$, and $P^{b}$ is the solution of Mckean-Vlasov equation,

$$
\frac{\partial}{\partial t} P^{b}=-\frac{\partial}{\partial x}\left[\alpha(m-x)+\omega(b-x) P^{b}\right]+\frac{1}{2} \sigma^{2} \frac{\partial^{2}}{\partial x^{2}} P^{b} .
$$

The steady state of $P^{b}$ is easy to calculate:

$$
P^{b}(x, \infty)=\frac{1}{Z} e^{-((\alpha+\omega) x-(\alpha m+\omega b) / \sigma)^{2}},
$$

where $Z$ is the normalization constant, so we have $P(\infty)=\int \mathrm{d} \mu(b) P^{b}(\infty)$.

Considering the limit behavior $\sigma \longrightarrow 0$, the steady state of Mckean-Vlasov equation when $h(y, x)=y-x$ has the form

$$
P(x, \infty)=\int \mathrm{d} \mu(b) \delta\left(x-\frac{\alpha m+\omega b}{\alpha+\omega}\right) .
$$

So that even in the case that the variance of the noise trends to 0 , this equation will not converge to a single Dirac measure, which is different from the $\omega=0$ case. Intuitively, the term $\omega(b-x)$ makes the opinion try not to deviate from the initial position too far, which can be considered as soft-bounded confidence.

In the case that $h(y, x)=\alpha y$, which is used in the SLANT system in the study by De et al. [9], supposing $\omega>\alpha$, the steady state will have the similar form since the equation of which is

$$
-\frac{\partial}{\partial x}\left[\alpha m+\omega(b-x) P^{b}\right]+\frac{1}{2} \sigma^{2} \frac{\partial^{2}}{\partial x^{2}} P^{b}=0,
$$

where $m=m(\infty)=(\alpha / \omega-\alpha) m(0)$ (this is from (25)), the final mean value of $x$. We have $P(\infty)=\int \mathrm{d} \mu(b) \delta(x-$ $(\alpha m+\omega b) / \omega)$.

4.4. Steady-State Distribution of Bounded Confidence Models. In the case that $\omega=0$ and $h(y, x)=(y-x) k(y-x)$, the steady-state distribution is studied [27]. We can follow their methods to study the weak solution of our equation. 


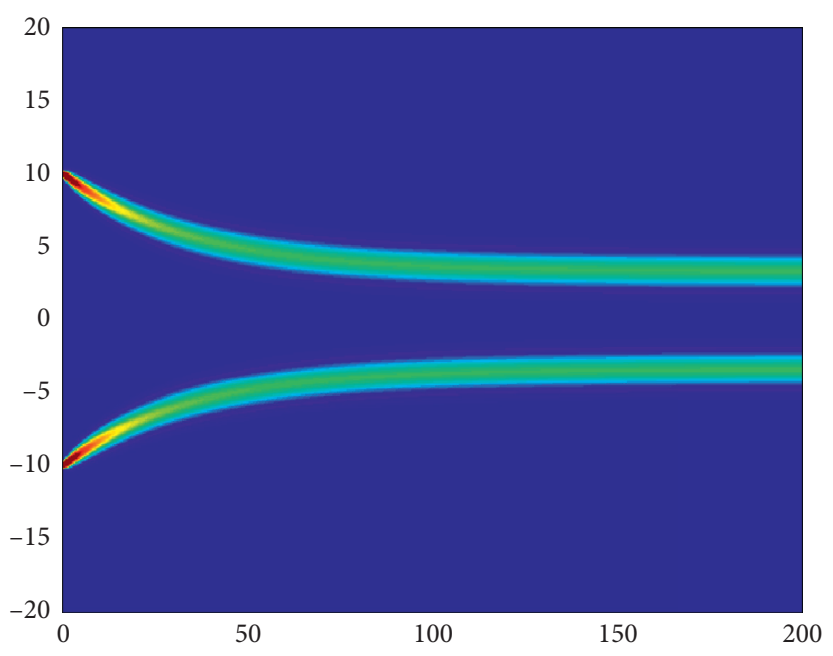

(a)

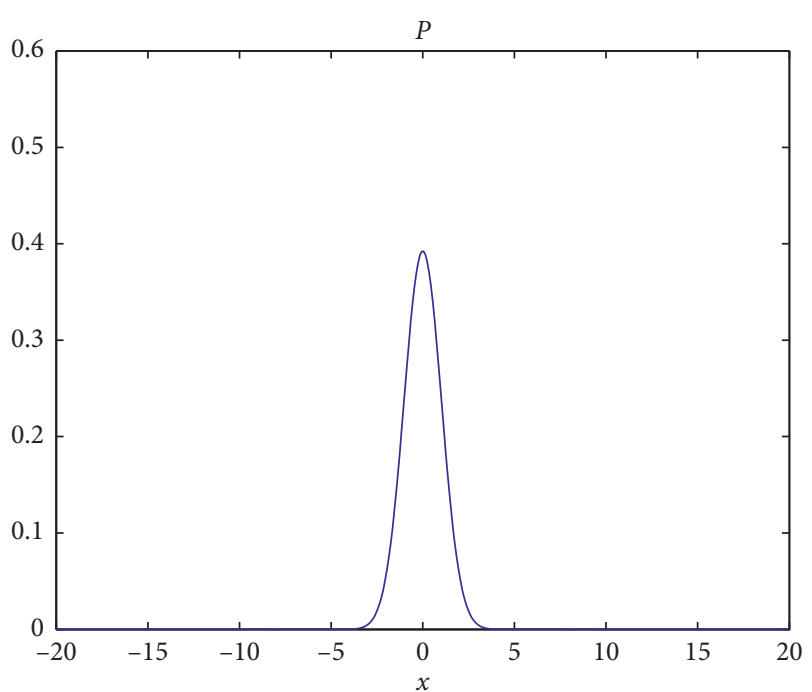

(b)

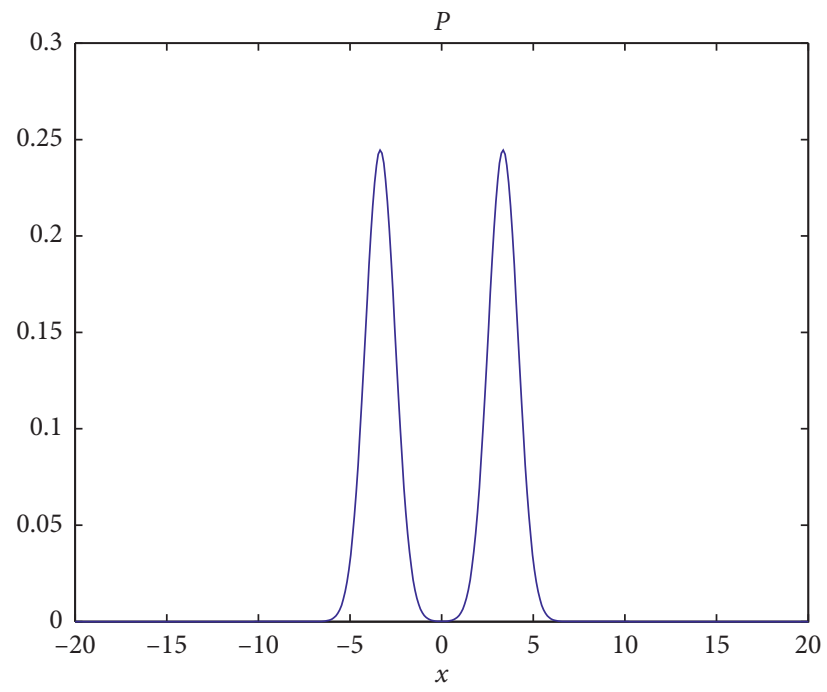

(c)

FIgURE 1: Simulation results. (a) Evolution of the opinion density, with $s=0: 02 ; a=0: 02 ; w=0: 01$. (b) Steady state for the dynamics with parameters $s=0: 02 ; a=0: 02 ; w=0$. (c) Solution profiles at time $t=200$, with parameters $s=0: 02 ; a=0: 02 ; w=0: 01$.

Theorem 4. Let $\omega=0, h(y, x)=(y-x) k(y-x)$, and $k(z)=0$ if $\|z\|>R$, otherwise $k(z)>0$. We have $P(x, \infty) \longrightarrow \sum_{i} C \delta\left(x-x_{i}\right)_{i} \quad$ when $\sigma \longrightarrow 0$, where $\left\|x_{i}-x_{j}\right\|>R$ if $i \neq j$.
Proof. Considering equation (19) with $\omega=0$, let $Q_{t}=\int \mu(b) x^{2} P^{b}(x)=\int x^{2} P(\mathrm{~d} x)$. We have

$$
\begin{aligned}
\frac{\mathrm{d}}{\mathrm{d} t} Q_{t} & =-\int \mu(\mathrm{d} b) x^{2} \frac{\partial}{\partial x} \int P(\mathrm{~d} y)\left[(y-x) k(y-x)+\omega(b-x) P^{b}(\mathrm{~d} x)\right]+\frac{1}{2} \int \sigma^{2} x^{2} \frac{\partial^{2}}{\partial x^{2}} P(\mathrm{~d} x) \\
& =\int 2 x(y-x) k(y-x) P(\mathrm{~d} y) P(\mathrm{~d} x) \frac{1}{2} \int \sigma^{2} x^{2} \frac{\partial^{2}}{\partial x^{2}} P(\mathrm{~d} x) \\
& \stackrel{(a)}{=}-\int(y-x)^{2} k(y-x) P(\mathrm{~d} y) P(\mathrm{~d} x)+\sigma^{2} \int P(\mathrm{~d} x),
\end{aligned}
$$


where $k(y-x)$ is a bounded confidence kernel. In (a) of (42), we use the symmetry of $P(x)$ and $P(y)$ and the integration by parts.

Considering the steady state and $\sigma \longrightarrow 0$ limit, we have $\mathrm{d} / \mathrm{d} t\left(Q_{t}\right)=0$, yet if in the support of $P(\infty)$, there are two points $x$ and $y$ such that $\|x-y\|<R$ and $x \neq y$, and we have $-\int(y-x)^{2} k(y-x) P(y) P(x)<0$, which is a contradiction, so that our claim follows.

Supposing $\omega>0$, it is hard to obtain a steady state directly. However, we can use $Q_{t}$ function to give an estimation. We can show that in the bounded confidence case, the steady-state solution is still a "contraction" of initial distribution.

$$
\begin{aligned}
\frac{\mathrm{d}}{\mathrm{d} t} Q_{t}= & -\int \mu(b) x^{2} \frac{\partial}{\partial x} \int P(\mathrm{~d} y)\left[(y-x) k(y-x) P^{b}(\mathrm{~d} x)\right]+\frac{1}{2} \int \sigma^{2} x^{2} \frac{\partial^{2}}{\partial x^{2}} P(\mathrm{~d} x)=\int 2 x(y-x) k(y-x) P(\mathrm{~d} y) P(\mathrm{~d} x) \\
& +\int 2 \omega x(b-x) \mu(\mathrm{d} b) P^{b}(\mathrm{~d} x)+\frac{1}{2} \int \sigma^{2} x^{2} \frac{\partial^{2}}{\partial x^{2}} P(\mathrm{~d} x)=-\int(y-x)^{2} k(y-x) P(\mathrm{~d} y) P(\mathrm{~d} x) \\
& +\int 2 \omega x(b-x) \mu(\mathrm{d} b) P^{b}(\mathrm{~d} x)+\sigma^{2} \int P(\mathrm{~d} x) .
\end{aligned}
$$

Since $-\int(y-x)^{2} k(y-x) P(\mathrm{~d} y) P(\mathrm{~d} x) \leq 0$ and $\sigma \longrightarrow 0$, we have

$$
\int x(b-x) \mu(\mathrm{d} b) P^{b}(\mathrm{~d} x) \geq 0,
$$

such that in the sense of mean value, $|x|<|b|=|x(0)|$. And the degree of offset is depending on the value of $\int(y-x)^{2} k(y-x) P(\mathrm{~d} y) P(\mathrm{~d} x)$. We can see that $\int(y-x)^{2}$ $k(y-x) P(\mathrm{~d} y) P(\mathrm{~d} x)$ will increase as the radius $R$ of the kernel $k(y-x)$ grows. When $R \longrightarrow \infty$ and $k(y-x)=$ 1 , the model will degenerate into the linear case in Theorem 3.

\section{The Outline of the Proof}

5.1. SDE Related to Initial Values. Since the process we study is not a Markov process, we need to consider SDE with the form

$$
\mathrm{d} X=F(X[0], X, t) \mathrm{d} t+\mathrm{d} W
$$

It has no Markov generator, but the non-Markovian of it is "not so bad". The law of $X$ can be solved by the following method. For a given $X[0]$, we can solve the law by $e^{t A} P_{0}$, where $A$ is the generator of the Fokker-Planck equation, and $P_{0}=\delta_{x_{0}}$ is the initial Dirac distribution. Then, the solution of the SDE has the form

$$
\int \mathrm{d} P_{0}\left(X_{0}\right) e^{t A\left(X_{0}\right)} \delta_{X_{0}}
$$

where $A\left(X_{0}\right)$ is the generator with given $X[0], \delta_{X_{0}}$ is the Dirac measure with the support on $X_{0}$, and $P_{0}$ is the initial distribution of $X_{0}$.

The essence of this method is to regard $X[0]$ as an additional random variable. When SDE has the above form, we need the double layer empirical measure, which will regain the symmetry.

We will consider the SDEs

$$
\mathrm{d} X_{i}=\omega\left(b_{i}-X_{i}(t)\right) \mathrm{d} t+\sum_{j \neq i} \alpha h\left(X_{j}, X_{i}\right) \mathrm{d} N_{j}+\sigma \mathrm{d} W_{i},
$$

where $b_{i}$ is a stochastic variable such that $X_{i}[0]=b_{i}$; then, we will prove the large number law for the double layer empirical measure $1 / N \sum_{i} \delta_{X_{i}, b_{i}}$, which will give the Mckean-Vlasov limit. This is equal to consider SDEs

$$
\mathrm{d} X_{i}^{b}=\omega\left(b-X_{i}^{b}(t)\right) \mathrm{d} t+\sum_{j \neq i} \int \mathrm{d} \mu\left(b^{\prime}\right) \alpha h\left(X_{j}^{b}, X_{i}^{b}\right) \mathrm{d} N_{j}+\sigma \mathrm{d} W_{i},
$$

where $X^{b}$ is the stochastic process with given $b$, and $E(X)=E\left(\int \mathrm{d} \mu(b) X^{b}\right)$.

5.2. Intermediate Process. Andreis et al. [15] proved that the Mckean-Vlasov limit of the equation

$$
\mathrm{d} X_{i}^{N}=F\left(X_{i}^{N}\right) \mathrm{d} t+\sigma\left(X_{i}^{N}\right) \mathrm{d} W_{i}+\frac{1}{N} \sum_{j \neq i}^{N} h\left(X_{j}^{N}, X_{i}^{N}\right) \mathrm{d} N_{j},
$$

has the form

$$
\mathrm{d} X=F(X)+\int \mathrm{d} P(Y) h(Y, X) \lambda \mathrm{d} t+\sigma(X) \mathrm{d} W,
$$

where $P$ is the law of $X$.

We use the same way as Andreis et al.[15] to reduce the jump-SDE to the "averaging dynamics." Their method is to decompose the jump terms into a martingale and a continuous part. Then, the martingale can be easily estimated by Burkholder-Davis-Gundy inequality.

We will show that there is an intermediate process $Y_{i}$ with the following SDE: 


$$
\mathrm{d} Y_{i}^{b}=\left(b-Y_{i}\right) \mathrm{d} t+\sigma \mathrm{d} W_{i}+\int \mathrm{d} \mu\left(b^{\prime}\right) \frac{1}{N} \sum_{j=1}^{N} \alpha h\left(Y_{j}^{b}, Y_{i}^{b}\right) \lambda \mathrm{d} t,
$$

such that

$$
\int d \mu(b) \frac{1}{N} \sum_{j=1}^{N} E\left[\sup \left\|X_{i}^{b}-Y_{i}^{b}\right\|\right] \leq \frac{C}{\sqrt{N}} .
$$
that

By comparing these two process directly, we can notice

$$
\int \mu(b) E\left[\sup _{r}\left\|X_{i}^{b}-Y_{i}^{b}\right\|\right] \leq \int \mu(b)(F+\Theta),
$$

where $F=\omega \int_{0}^{t}\left\|X_{i}^{b}(s)-Y_{i}^{b}(s)\right\| \mathrm{d} s$, and $\Theta=E\left[\sup _{r} \| 1 /\right.$ $\left.N \sum_{j} \int_{0}^{r} \mathrm{~d} s\left(\alpha h\left(X_{j}, X_{i}\right) \mathrm{d} N_{j}(s)-\alpha h\left(Y_{j}, Y_{i}\right) \lambda \mathrm{d} s\right) \|\right]$.

Using Doob-Meyer decomposition,

$$
\mathrm{d} N=\mathrm{d} \tilde{N}+\lambda \mathrm{d} t,
$$

where $\widetilde{N}$ is a martingale.

Intuitively, the supremum of a martingale would not be very large. This can be proved by Burkholder-Davis-Gundy inequality and our assumption on $h$. Then, we can obtain the approximation (52). See the second part of the appendix for a detailed proof of (52).

5.3. Mckean-Vlasov Process. The intermediate process has the form like

$$
\mathrm{d} X_{i}=\frac{1}{N} \sum_{j} f\left(X_{i}, X_{j}\right) \mathrm{d} t+g\left(X_{i}, \omega_{i}\right) \mathrm{d} t+\mathrm{d} W,
$$

where $\omega_{i}$ is a random variable.

The asymptotic behavior of the double layer empirical measure, with the case that $f$ and $g$ are bounded, has been studied [38]. They use Varadhan's lemma to reduce the system to the case without interaction. Since $f$ and $g$ are not bounded, we use the method in the study by Sznitman [28] to compare the two SDEs directly. Using the Lipschitz condition, we can control $E\left[\sup _{r}\|X(r)-\bar{X}(r)\|\right]$ by the first Wasserstein distance $\rho$.

The first Wasserstein distance is defined by

$$
W_{1}\left(\mu_{1}, \mu_{2}\right)=\inf \int_{R^{d} \times R^{d}}\left|x-y \eta(\mathrm{d} x, \mathrm{~d} y): \eta \in H\left(\mu_{1}, \mu_{2}\right)\right| \text {, }
$$

where $H\left(\mu_{1}, \mu_{2}\right)$ is the set of all probability measures on $R^{d} \times$ $R^{d}$ with marginals $\mu_{1}$ and $\mu_{2}$.

By Kantorovich-Rubinstein duality theorem, it has a dual representation:

$$
\sup _{\|f\|_{L} \leq 1} \int f(x) \mathrm{d}\left(\mu_{1}-\mu_{2}\right)(x),
$$

where $\|f\|_{L}$ is the minimal Lipschitz constant for $f$.

Using this representation, since we have assumed that the coefficients are Lipschitz, we can see that

$$
E\left[\sup _{r}\|X(r)-\bar{X}(r)\| \leq L \int_{0}^{T} E\left[\rho\left(\mu_{X}^{N}(t), \mu_{t}\right)\right]\right] \mathrm{d} t,
$$

where $\mu_{X}^{N}$ is the empirical measure, and $\mu_{t}$ is the solution of Mckean-Vlasov equation.

For $E\left[\rho\left(\mu_{X}^{N}(t), \mu_{t}\right)\right]$, we have

$$
E\left[\rho\left(\mu_{X}^{N}(t), \mu_{t}\right)\right] \leq E\left[\rho\left(\mu_{X}^{N}(t), \mu_{\bar{X}}^{N}(t)\right)\right]+E\left[\rho\left(\mu_{\bar{X}}^{N}(t), \mu_{t}^{N}\right)\right],
$$

where $\mu \frac{N}{\bar{X}}$ is the empirical measure for $N$ independent Mckean-Vlasov processes.

Then, we can derive our result by considering the Wasserstein distance between the solution of Mckean-Vlasov equation and empirical measure. Using Theorem 1 in the study by Zanella and Guillin [41], if the two order moment is finite,

$$
E\left[\bar{X}^{2}\right]<\infty
$$

when the size tends to infinity and the Wasserstein distance trends to zero, so our claim follows. The detailed proof is given in the appendix.

\section{Conclusion}

In this paper, we studied the asymptotic behaviors of the exponential function weight of the self-excitation interaction system $X^{N}$ on the large homogeneous network when $N$, the number of the individuals, trends to infinity. We proved that there is a Mckean-Vlasov process $\bar{X}$, such that $\lim _{N \longrightarrow+\infty} E\left|X^{N}-\bar{X}\right|$ is zero, whose opinion distribution evolve according to a Mckean-Vlasov type integrodifferential equation, which couples with the initial distribution. The steady state of this equation is also studied. We investigated the steady state distribution of linear and bounded confidence models and showed that the steady distribution of these models is a "contraction" of initial distribution. By virtue of the self-excitation interaction, even if we do not consider bounded confidence, it will give not a perfect consensus, but a distribution depending on the initial distribution, such as this model, can avoid Abelson's diversity puzzle.

\section{Appendix}

\section{A. The Condition under which the Assumptions Hold}

In this section, we will give some examples that the assumptions can be satisfied. We will show that when $h$ has linear growth, the assumptions (16) and (17) are satisfied.

For SDE,

$$
\mathrm{d} X_{i}^{b}=\frac{1}{N} \sum_{j \neq i} \int \mathrm{d} \mu\left(b^{\prime}\right) \alpha h\left(X_{j}^{b}, X_{i}^{b}\right) \mathrm{d} N_{j}+\omega\left(b-X_{i}^{b}\right) \mathrm{d} t+\sigma \mathrm{d} W_{i},
$$


and it is shown in the study by Situ [42] that since both $b-X$ and $h$ has linear growth, this SDE has a strong solution, and also, we can estimate the moment.
In order to prove (16), since $h$ is linear growth, we can turn to consider the second moment by Jensen's inequality $E[h(y, x)] \leq L(E[|y|]+E[|x|]) \leq 2 L\left(E\left[x^{2}\right]\right)^{1 / 2}$. Using Ito formula,

$$
\begin{aligned}
\int \mathrm{d} \mu(b) \frac{1}{N} \sum_{i}\left(X_{i}^{b}(T)\right)^{2}= & \int \mathrm{d} \mu(b)\left[\frac{1}{N} \sum_{i}\left(X_{i}^{b}(0)^{2}\right)+\int_{0}^{T} X_{i}^{b}(s) b-\left(X_{i}^{b}(s)^{2} \mathrm{~d} s\right)\right] \\
& +\int \mathrm{d} \mu(b) \mathrm{d} \mu\left(b^{\prime}\right) \frac{1}{N}\left[\sum_{i, j} \int_{0}^{T} 2 X_{i}^{b}(s) \frac{1}{N} \alpha h\left(X_{j}^{b}, X_{i}^{b}\right)+\frac{1}{N^{2}} \alpha^{2}\left|h\left(X_{j}^{b}, X_{i}^{b}\right)\right|^{2} \mathrm{~d} N_{j}\right]+C+\text { martingale. }
\end{aligned}
$$

Since $\quad|h(x, y)| \leq L(|X|+|Y|) \quad$ and $\quad \mathrm{d} N=\lambda \mathrm{d} t+$ martingale, we can show that

$$
\begin{aligned}
\int \mathrm{d} \mu(b) \frac{1}{N} \sum_{i} E\left(X_{i}^{b}(T)\right)^{2} \leq & \int \mathrm{d} \mu(b) \frac{1}{N} \sum_{i}\left[X_{i}^{b}(0)^{2}\right. \\
& +K \int_{0}^{T} E\left(X_{i}^{b}(s)\right)^{2}+C \mathrm{~d} s \\
& \left.+\int_{0}^{T} E\left(\left(X_{i}^{b}(s)\right) b-E\left(X_{i}^{b}(s)\right)^{2} \mathrm{~d} s\right)\right] .
\end{aligned}
$$

We use $E[X] \leq E[|X|] \leq\left(E\left[X^{2}\right]\right)^{1 / 2} \leq K\left(1+E\left[X^{2}\right]\right)$ by Jensen's inequality. Then, using Gronwall' lemma, our claim follows.

As for the second condition (17), the above method can be also applicable.

\section{B. Reduce to the Averaging Dynamics}

The SDE for stochastic process $X_{i}$ is

$$
\mathrm{d} X_{i}^{b}=g\left(b, X_{i}^{b}\right) \mathrm{d} t+\sigma \mathrm{d} W_{i}+\int \mathrm{d} \mu\left(b^{\prime}\right) \frac{1}{N} \sum_{j \neq i}^{N} \alpha h\left(X_{j}^{b}, X_{i}^{b}\right) \mathrm{d} N_{j} .
$$

Following the study by Andreis et al. [15], it is useful to introduce an intermediate process $Y_{i}$ with SDE

$$
\mathrm{d} Y_{i}^{b}=g\left(b, Y_{i}^{b}\right) \mathrm{d} t+\sigma \mathrm{d} W_{i}+\int \mathrm{d} \mu\left(b^{\prime}\right) \frac{1}{N} \sum_{j=1}^{N} \alpha h\left(Y_{j}^{b}, Y_{i}^{b}\right) \lambda \mathrm{d} t
$$

Since the Poisson process is a semimartingale, $N(t)$ can be decomposed into $N(t)=\tilde{N}(t)+\lambda t$ by Doob-Meyer decomposition, where $\tilde{N}(t)$ is a martingale. The $\lambda \mathrm{d} t$ in $Y$ is the second part of Doob-Meyer decomposition of $N$. To simplify the symbol, we set $\lambda=1$.

We will show that for large $N$,

$$
\int \mathrm{d} \mu(b) \frac{1}{N} \sum_{j=1}^{N} E\left[\sup _{r \in[0, t]}\left\|X_{j}^{b}(r)-Y_{j}^{b}(r)\right\|\right] \leq \frac{C}{\sqrt{N}}
$$

so, $X$ can be approximated by $Y$.

Let

$$
\begin{aligned}
& G_{i}^{b}=E\left[\int_{0}^{t}\left\|g\left(X_{i}^{b}, b\right)-g\left(Y_{i}^{b}, b\right)\right\| \mathrm{d} s\right], \\
& \Theta_{i}=E\left[\sup _{r}\left\|\mathrm{~d} \mu\left(b^{\prime}\right)\left[\frac{1}{N} \int \sum_{j \neq i} \int_{0}^{r} \alpha h\left(X_{j}^{b}, X_{i}^{b}\right) \mathrm{d} N_{j}-\frac{1}{N} \sum_{j} \int_{0}^{r} \alpha h\left(Y_{j}^{b}, Y_{i}^{b}\right) \mathrm{d} s\right]\right\|\right],
\end{aligned}
$$


so,

$$
\begin{aligned}
& \int \mathrm{d} \mu(b) \frac{1}{N} \sum_{i} E\left[\sup _{r}\left\|X_{i}^{b}(r)-Y_{i}^{b}(r)\right\|\right] \\
& \leq \int \mathrm{d} \mu(b)\left[\frac{1}{N} \sum_{i}\left(G_{i}+\Theta_{i}\right)\right] .
\end{aligned}
$$

In our model $(9), g\left(x_{i}, b\right)=\omega\left(b-x_{i}\right)$, so that we have $G_{i}=E\left[\int_{0}^{t}\left\|\omega\left(Y_{i}^{b}-X_{i}^{b}\right)\right\|\right]$.

For $\Theta$,

$$
\begin{aligned}
\Theta_{i} \leq & E\left[\sup _{r}\left\|\int \mathrm{d} \mu\left(b^{\prime}\right) \frac{1}{N} \sum_{j \neq i} \int_{0}^{r} \alpha h\left(X_{j}^{b}, X_{i}^{b}\right) \mathrm{d} \tilde{N}(t)\right\|\right]+E\left[\sup _{r}\left\|\int \mathrm{d} \mu\left(b^{\prime}\right) \frac{1}{N} \sum_{j} \int_{0}^{r} \alpha\left(h\left(X_{j}^{b}, X_{i}^{b}\right)-h\left(Y_{j}^{b}, Y_{i}^{b}\right)\right) \mathrm{d} s\right\|\right] \\
& +\frac{1}{N} E\left[\sup _{r}\left\|\int_{0}^{r} \int \mathrm{d} \mu\left(b^{\prime}\right) \alpha h\left(X_{i}^{b}, X_{i}^{b}\right) \mathrm{d} s\right\|\right] .
\end{aligned}
$$

As in the study by Andreis et al. [15], we can use the Burkholder-Davis-Gundy inequality for martingales.

$$
\begin{aligned}
\Theta_{i} & \leq \frac{k}{N} E\left[\left(\sum_{j \neq i} \int_{0}^{t}\left\|\int \mathrm{d} \mu\left(b^{\prime}\right) h\left(X_{j}^{b}, X_{i}^{b}\right)\right\|^{2} \mathrm{~d} s\right)^{1 / 2}\right]+\frac{1}{N} \int_{0}^{t} E\left[\sup \sum_{j} \alpha\left\|\int \mathrm{d} \mu\left(b^{\prime}\right) h\left(X_{j}^{b}, X_{i}^{b}\right)-h\left(Y_{j}^{b}, Y_{i}^{b}\right)\right\|\right] \mathrm{d} s+\frac{H}{N} \\
& \leq \frac{k}{N} \int_{0}^{t} E\left[N\left(\left\|\int \mathrm{d} \mu\left(b^{\prime}\right) h\left(X_{j}^{b}, X_{i}^{b}\right)\right\|^{2} \mathrm{~d} s\right)^{1 / 2}\right]+\frac{1}{N} \int_{0}^{t} E\left[\sum_{j} \alpha\left\|\int \mathrm{d} \mu\left(b^{\prime}\right) h\left(X_{j}^{b}, X_{i}^{b}\right)-h\left(Y_{j}^{b}, Y_{i}^{b}\right)\right\|\right] \mathrm{d} s+\frac{H}{N} \\
& \leq \frac{k}{N} E\left[\left(N t\left(\sup _{r}\left\|\int \mathrm{d} \mu\left(b^{\prime}\right) h\left(X_{j}^{b}(r), X_{i}^{b}(r)\right)\right\|\right)^{2}\right)^{1 / 2}\right]+\int_{0}^{t} \frac{1}{N} E\left[\sum_{j} \alpha\left\|\int \mathrm{d} \mu\left(b^{\prime}\right) h\left(X_{j}^{b}, X_{i}^{b}\right)-h\left(Y_{j}^{b}, Y_{i}^{b}\right)\right\|\right] \mathrm{d} s+\frac{H}{N} \\
& \leq \frac{C}{\sqrt{N}}+L \int_{0}^{t} E\left[\left\|X_{i}^{b}-Y_{i}^{b}\right\|\right] \mathrm{d} s+\frac{L}{N} \sum_{j} \int \mathrm{d} \mu\left(b^{\prime}\right) \int_{0}^{t} E\left[\left\|X_{j}^{b}-Y_{j}^{b}\right\|\right] \mathrm{d} s+\frac{H}{N},
\end{aligned}
$$

where we use the symmetry, assumption (16), and the Lipschitz condition for $h$. Combine the two results; then, we get

$$
\begin{aligned}
\int \mathrm{d} \mu(b) \frac{1}{N} \sum_{j} E\left[\sup \left\|X_{i}^{b}-Y_{i}^{b}\right\|\right] \leq & \frac{C}{\sqrt{N}}+K \int \mathrm{d} \mu(b) \frac{1}{N} \sum_{j} \int_{0}^{t} E\left[\left\|X_{i}^{b}-Y_{i}^{b}\right\|\right] \mathrm{d} s \\
+\frac{H}{N} & \leq \frac{C}{\sqrt{N}}+K \int \mathrm{d} \mu(b) \frac{1}{N} \sum_{j} \int_{0}^{t} E\left[\sup \left\|X_{i}^{b}-Y_{i}^{b}\right\|\right] \mathrm{d} s+\frac{H}{N} .
\end{aligned}
$$


Then, using the standard technology of Gronwall's lemma, we get the approximation.

\section{Mckean-Vlasov process}

As shown in the previous section, we can turn to analyze the stochastic process $Y$. Let $\bar{X}$ be a stochastic process with SDE:

$$
\mathrm{d} \bar{X}=\int \mathrm{d} P(y) \alpha h(y, \bar{X}) \mathrm{d} t+\omega(b-\bar{X}) \mathrm{d} t+\sigma \mathrm{d} W,
$$

where $P$ is the law of $\bar{X}$, and $b$ is a random variable, such that $b=X[0]$.
Using the trick in (47), we can show that

$$
P(y)=\int \mathrm{d} \mu(b) P^{b}(y),
$$

where $P^{b}$ is the law of SDE with given $b$, the initial distribution is $\delta_{b}$, and $\mu(b)$ is the given initial distribution of $\bar{X}$. We will show that

$$
\lim _{N \longrightarrow \infty} \int \mathrm{d} \mu(b) \frac{1}{N} \sum_{i} E\left[\sup _{t \leq T}\left|Y_{t}^{b, i}-\bar{X}_{t}^{b, i}\right|\right]=0 .
$$

Let $Q^{N}$ be the empirical measures $\sum_{j}(1 / N) \delta_{\overline{X_{j}}}$, we have

$$
\begin{aligned}
Y_{t}^{b, i}-\bar{X}_{t}^{b, i}= & \int_{0}^{t} \frac{1}{N} \int \mathrm{d} \mu\left(b^{\prime}\right) \sum_{j} h\left(Y_{s}^{b, i}, Y_{s}^{b^{\prime}, j}\right)-\int h\left(\bar{X}_{s}^{b, i}, y\right) Q(\mathrm{~d} y) \mathrm{d} s+\omega \int_{0}^{t} \bar{X}_{s}^{b, i}-Y_{s}^{b, i} \mathrm{~d} s \\
= & \int_{0}^{t} \mathrm{~d} s \frac{1}{N} \int \mathrm{d} \mu\left(b^{\prime}\right) \sum_{j} h\left(Y_{s}^{b, i}, Y_{s}^{b^{\prime}, j}\right)-h\left(\bar{X}_{s}^{b, i}, Y_{s}^{b, j}\right)+\left(h\left(\bar{X}_{s}^{b, i}, Y_{s}^{b, j}\right)-h\left(\bar{X}_{s}^{b, i}, \bar{X}_{s}^{b, j}\right)\right) \\
& +\frac{1}{N} \sum_{j} \int \mathrm{d} \mu\left(b^{\prime}\right)\left(h\left(\bar{X}_{s}^{b, i}, \bar{X}_{s}^{b, j}\right)-\int h\left(\bar{X}_{s}^{b, i}, y\right) Q(\mathrm{~d} y)\right)+\omega \int_{0}^{t} \bar{X}_{s}^{b, i}-Y_{s}^{b, i} \mathrm{~d} s .
\end{aligned}
$$

Since $h$ is Lipschitz, we can see that

$$
\begin{aligned}
& \int \mathrm{d} \mu(b) E\left[\left|\sup _{r} Y^{b, i}(r)-\bar{X}^{b, i}(r)\right|\right] \leq L \int \mathrm{d} \mu(b) \sup _{r} \int_{0}^{r} \mathrm{~d} s\left(E\left[\left|Y^{b, i}-\bar{X}^{b, i}\right|\right]\right)+\frac{1}{N} \sum_{j} \int \mathrm{d} \mu\left(b^{\prime}\right) E\left[\left|Y^{b^{\prime}, j}-\bar{X}^{b^{\prime}, j}\right|\right] \\
& +\int \mathrm{d} \mu(b) E \mid \frac{1}{N} \sum_{j} \int \mathrm{d} \mu\left(b^{\prime}\right) h\left(\bar{X}^{b, i}, \bar{X}^{b^{\prime}, j}\right)-\int h\left(\bar{X}^{b, i}, y\right) Q(\mathrm{~d} y)+\omega \int_{0}^{t}\left\|\bar{X}_{s}^{b, i}-Y_{s}^{b, i}\right\| .
\end{aligned}
$$

Summing over $i$,

$$
\begin{aligned}
& \frac{1}{N} \sum_{i} \int \mathrm{d} \mu(b) E\left[\sup _{r}\left|Y^{b, i}(r)-\bar{X}^{b, i}(r)\right|\right] \\
& \leq \frac{1}{N}\left\{L \sup _{r} \int_{0}^{r} \mathrm{~d} s \sum_{i} \int \mathrm{ds}(b)\left[E\left[|| Y^{b, i}-\bar{X}^{b, i} \mid\right]\right]+E \mid \frac{1}{N} \sum_{j} \int \mathrm{d} \mu\left(b^{\prime}\right) h\left(\bar{X}^{b, i}, \bar{X}^{b^{\prime}, j}\right)-\int h\left(\bar{X}^{b, i}, y\right) Q(\mathrm{~d} y)+\omega \int_{0}^{t} \sum_{i} E\left\|\bar{X}_{s}^{b, i}-Y_{s}^{b, i}\right\|\right\}
\end{aligned}
$$

then, using Gronwall's lemma

$$
\begin{aligned}
& \frac{1}{N} \sum_{i} \int \mathrm{d} \mu(b) E\left[\sup _{r}\left|Y^{b, i}(r)-\bar{X}^{b, i}(r)\right|\right] \\
& \left.\leq L(T) \sup _{r} \int_{0}^{r} \int \mathrm{d} \mu(b) E \mid \frac{1}{N} \sum_{j} \int \mathrm{d} \mu\left(b^{\prime}\right) h\left(\bar{X}^{b, i}, \bar{X}^{b^{\prime}, j}\right)-\int h\left(\bar{X}^{b, i}, y\right) Q(\mathrm{~d} y)\right] \mathrm{d} s .
\end{aligned}
$$


So, we only need to estimate the right side.

Using the Lipschitz condition for $h$ again, this can be controlled by $E\left[\rho\left[\left(Q_{\bar{X}}^{N}(t), Q_{\bar{X}}(t)\right)\right]\right]$ where $\rho$ is the Wasserstein distance between the two measures, and $Q_{\bar{X}}$ is the solution of Mckean-Vlasov equation (72). $Q^{N}$ is the empirical measures $\sum_{j}(1 / N) \delta_{\overline{X_{j}}}$. It follows from [41] Theorem 1 that if the moments is bounded (this is satisfied by (17))

$$
E\left[\bar{X}^{2}\right]<\infty \text {. }
$$

Thus, our claim follows.

\section{Data Availability}

This paper is not data-driving, and the model we study is from other data-driving ones. The data used in this article are only the data of the simulation of PDE, which are contained in Figure 1.

\section{Conflicts of Interest}

The authors declare that they have no conflicts of interest.

\section{Acknowledgments}

The authors thank Wenyu Zhang for his great help in writing and Prof. Haibo Wang who introduced the recent developments in the mean-field game theory to us. This research was funded by the National Key Research and Development Program of China, grant number 2018YFC0831300.

\section{References}

[1] T. Karppi and K. Crawford, "Social media, financial algorithms and the hack crash," Theory, Culture \& Society, vol. 33, no. 1, pp. 73-92, 2016.

[2] A. Ceron, L. Curini, and S. M. Iacus, "Using sentiment analysis to monitor electoral campaigns," Social Science Computer Review, vol. 33, no. 1, pp. 3-20, 2015.

[3] K. Sznajd-Weron and J. Sznajd, "Opinion evolution in closed community," International Journal of Modern Physics C, vol. 11, no. 6, pp. 1157-1165, 2000.

[4] R. Hegselmann and U. Krause, "Opinion dynamics driven by various ways of averaging," Computational Economics, vol. 25, no. 4, pp. 381-405, 2005.

[5] R. Hegselmann and U. Krause, "Opinion dynamics and bounded confidence models, analysis and simulation," Journal of Artificial Societies \& Social Simulation, vol. 5, no. 3, p. 2, 2002.

[6] G. r. Weisbuch, G. Deffuant, F. d. r. Amblard, and J.-P. Nadal, "Meet, discuss, and segregate!" Complexity, vol. 7, no. 3, pp. 55-63, 2002.

[7] A. Das, S. Gollapudi, and K. Munagala, "Modeling opinion dynamics in social networks," in Proceedings of the ACM International Conference on Web Search and Data Mining, pp. 403-412, New York, NY, USA, 2014.

[8] A. De, S. Bhattacharya, P. Bhattacharya, N. Ganguly, and S. Chakrabarti, "Learning a linear influence model from transient opinion dynamics," in Proceedings of the ACM International Conference on Conference on Information and Knowledge Management, pp. 401-410, Shanghai, China, 2014.
[9] A. De, I. Valera, N. Ganguly, S. Bhattacharya, and M. G. Rodriguez, Learning and Forecasting Opinion Dynamics in Social Networks, 2015, http://arxiv.org/abs/1506.05474v3.

[10] K. Zhou, H. Zha, and L. Song, "Learning social infectivity in sparse low-rank networks using multi-dimensional hawkes processes," in Proceedings of Machine Learning Research, PMLR, C. M. Carvalho and P. Ravikumar, Eds., in Proceedings of the Sixteenth International Conference on Artificial Intelligence and Statistics, vol. 31, pp. 641-649pp. 641-, Scottsdale, AZ, USA, 2013.

[11] Y. Wang, E. Theodorou, A. Verma, and L. Song, "A stochastic differential equation framework for guiding online user activities in closed loop," in Proceedings of Machine Learning Research, PMLR, A. Storkey and F. Perez-Cruz, Eds., in Proceedings of the Twenty-First International Conference on Artificial Intelligence and Statistics, vol. 84, pp. 10771086pp. 1077-, Playa Blanca, Spain, 2018.

[12] Y. Wang, G. Williams, E. Theodorou, and L. Song, "Variational policy for guiding point processes," in Proceedings of the International Conference on Machine Learning, pp. 36843693, Sydney, Australia, 2017.

[13] Y. Wang, X. Ye, H. Zha, and L. Song, "Predicting user activity level in point processes with mass transport equation," in Advances in Neural Information Processing Systems, I. Guyon, U. V. Luxburg, S. Bengio et al., Eds., vol. 30, pp. 1645-1655, Curran Associates, Inc., Red Hook, NY, USA, 2017.

[14] S. Méléard, "Asymptotic behaviour of some interacting particle systems; McKean-Vlasov and Boltzmann models," in Probabilistic Models for Nonlinear Partial Differential Equations, pp. 42-95, Springer, Berlin, Germany, 1996.

[15] L. Andreis, P. D. Pra, and M. Fischer, "McKean-Vlasov limit for interacting systems with simultaneous jumps," 2017, http://arxiv.org/abs/1704.01052v1.

[16] G. Toscani, "Kinetic models of opinion formation," Communications in Mathematical Sciences, vol. 4, no. 3, pp. 481496, 2006.

[17] A. T. Bernardes, D. Stauffer, and J. Kertész, "Election results and the Sznajd model on Barabasi network," The European Physical Journal B, vol. 25, no. 1, pp. 123-127, 2002.

[18] D. Stauffer, "Better being third than second in a search for a majority opinion," Advances in Complex Systems, vol. 5, no. 1, pp. 97-100, 2002.

[19] D. Stauffer and P. M. C. de Oliveira, "Persistence of opinion in the Sznajd consensus model: computer simulation," The European Physical Journal B-Condensed Matter, vol. 30, no. 4, pp. 587-592, 2002.

[20] F. Slanina and H. Lavicka, "Analytical results for the Sznajd model of opinion formation," The European Physical Journal B-Condensed Matter, vol. 35, no. 2, pp. 279-288, 2003.

[21] J. Zhang, G. Chen, Y. Hong, J. Zhang, G. Chen, and Y. Hong, "Convergence analysis of asymmetric homogeneous deffuantweisbuch model," in Proceedings of the China Conference on Control and Decision-Making, pp. 2394-2399, Taiyuan, China, 2012.

[22] A. Bhattacharyya, M. Braverman, B. Chazelle, and H. L. Nguyen, "On the convergence of the hegselmann-krause system," in Proceedings of the 4th Conference on Innovations in Theoretical Computer Science, ITCS'13, pp. 61-66, ACM, New York, NY, USA, 2013.

[23] L. Guo and X. Cai, "Bifurcation phenomena of opinion dynamics in complex networks," in Proceedings of the International Conference on Complex Sciences, pp. 1146-1153, Shanghai, China, 2009. 
[24] Y. Liu, S.-M. Diao, Y.-X. Zhu, and Q. Liu, "SHIR competitive information diffusion model for online social media," Physica A: Statistical Mechanics and its Applications, vol. 461, pp. 543-553, 2016.

[25] F. Xiong, W. Shen, H. Chen, S. Pan, X. Wang, and Z. Yan, "Exploiting implicit influence from information propagation for social recommendation," IEEE Transactions on Cybernetics, 2019.

[26] Z. Li, F. Xiong, X. Wang, H. Chen, and X. Xiong, "Topological influence-aware recommendation on social networks," Complexity, vol. 2019, Article ID 6325654, 12 pages, 2019.

[27] G. Como and F. Fagnani, "Scaling limits for continuous opinion dynamics systems," The Annals of Applied Probability, vol. 21, no. 4, pp. 1537-1567, 2011.

[28] A. S. Sznitman, "Topics in propagation of chaos," Biophysical Journal, vol. 108, no. 2, p. 501, 1991.

[29] G. Albi, L. Pareschi, and M. Zanella, "Opinion dynamics over complex networks: kinetic modeling and numerical methods," Kinetic \& Related Models, vol. 10, no. 1, pp. 1-32, 2016.

[30] B. J. Cho, A Simulation Study on Interference in CSMA/CA Ad-Hoc Networks Using Point Process, Aalto University, Helsinki, Finland, 2010.

[31] V. Chavez-Demoulin and J. A. Mcgill, "High-frequency financial data modeling using Hawkes processes," Journal of Banking \& Finance, vol. 36, no. 12, pp. 3415-3426, 2012.

[32] F. Deschatres and D. Sornette, "Dynamics of book sales: endogenous versus exogenous shocks in complex networks," Physical Review E, vol. 72, no. 2, Article ID 016112, 2005.

[33] R. Crane and D. Sornette, "Robust dynamic classes revealed by measuring the response function of a social system," Proceedings of the National Academy of Sciences, vol. 105, no. 41, pp. 15649-15653, 2008.

[34] J.-D. Deuschel and D. W. Stroock, "Large deviations,"vol. 342American Mathematical Society, Providence, RI, USA, 2001.

[35] G. B. Arous and A. Guionnet, "Large deviations for Langevin spin glass dynamics," Probability Theory and Related Fields, vol. 102, no. 4, pp. 455-509, 1995.

[36] D. A. Dawsont and J. Gärtner, "Large deviations from the Mckean-Vlasov limit for weakly interacting diffusions," Stochastics, vol. 20, no. 4, pp. 247-308, 1987.

[37] A. Puhalskii, Large Deviations and Idempotent Probability, Chapman \& Hall/CRC, London, UK, 2001.

[38] P. D. Pra and F. D. Hollander, "McKean-Vlasov limit for interacting random processes in random media," Journal of Statistical Physics, vol. 84, no. 3-4, pp. 735-772, 1996.

[39] R. P. Abelson, "Mathematical models of the distribution of attitudes under controversy," in Contributions to Mathematical Psychology, N. Frederiksen and H. Gulliken, Eds., Holt McDougal, New York, NY, USA, 1964.

[40] G. Albi, L. Pareschi, and M. Zanella, "Opinion dynamics over complex networks: kinetic modelling and numerical methods," Kinetic \& Related Models, vol. 10, no. 1, pp. 1-32, 2017.

[41] N. Zanella and A. Guillin, "On the rate of convergence in Wasserstein distance of the empirical measure," Probability Theory \& Related Fields, vol. 36, no. 3, pp. 1-32, 2013.

[42] R. Situ, Theory of Stochastic Differential Equations with Jumps and Applications: Mathematical and Analytical Techniques with Applications to Engineering, Springer Science \& Business Media, Berlin, Germany, 2006. 